\title{
The Stochastic Frontier Analysis Technique in Measuring the Technical and Economic Efficiency of Hospital Diagnostic Laboratories: A Case Study in Iran
}

\author{
Siamak Aghlmand ${ }^{1}$, Sadegh Feizollahzadeh ${ }^{2}$, hasan yusefzadeh ${ }^{3}$, and Mina Alinejhad ${ }^{2}$ \\ ${ }^{1}$ Urmia University of Medical Sciences School of Health and Paramedicine \\ ${ }^{2}$ Urmia University of Medical Sciences \\ ${ }^{3}$ Affiliation not available
}

June 9, 2020

\begin{abstract}
Rationale, aims, and objectives An inefficient health system wastes scarce resources even if it makes considerable gains in accountability and equity. Such a system is expected to perform better. Therefore, it is vital to examine the current performance of health systems and their constituents as well as assess how to reach their full potential. The aim of this study was to evaluate the technical and economic efficiency of medical diagnostic laboratories in hospitals affiliated with Urmia Medical Sciences University (UMSU) in 2016. Methods In this descriptive-analytical study, the data of all diagnostic laboratories of the hospitals of UMSU were inputted into Frontier 4.1 software after taking the logarithm. Then, the technical and economic efficiency of the laboratories were obtained by estimating the production and cost function using the stochastic frontier analysis method, assuming input minimization for 2016. Results The average technical and economic efficiency of the diagnostic laboratories was obtained as $0.931 \pm 0.34$ and $0.519 \pm 0.33$, respectively. These laboratories must reduce their inputs and costs to achieve full efficiency without changing the amount of their output. Conclusion Although the average economic efficiency of the diagnostic laboratories of the studied hospitals was high, there is still an increase in the efficiency of these units given the cost of inputs at the time of allocating resources. In addition, it is possible to improve the technical efficiency of the clinical laboratories of hospitals affiliated with UMSU by $48.1 \%$ by applying the same level of inputs and without increasing the costs.
\end{abstract}

The Stochastic Frontier Analysis Technique in Measuring the Technical and Economic Efficiency of Hospital Diagnostic

\section{Laboratories: A Case Study in Iran}

Siamak Aghlmand ${ }^{1}$, Sadegh Feizollahzadeh ${ }^{2}$, Mina Alinejhad ${ }^{1}$, Hasan Yusefzadeh ${ }^{1}$

${ }^{1}$ Health Economics and Management Department, School of Public Health, Urmia University of Medical Sciences, Urmia, Iran

${ }^{2}$ Laboratory Sciences Department, School of Allied Medical Sciences, Urmia University of Medical Sciences, Urmia, Iran

\section{Correspondence:}

Hasan Yusefzadeh, Iran, Urmia, Sero Street, Nazloo Paradise, School of Public Health, Postal Code: 5756115198, Tel: +9832752300. Fax: +9832752309. E-mail: hyusefzade2010@gmail.com

Rationale, aims, and objectives 
An inefficient health system wastes scarce resources even if it makes considerable gains in accountability and equity. Such a system is expected to perform better. Therefore, it is vital to examine the current performance of health systems and their constituents as well as assess how to reach their full potential. The aim of this study was to evaluate the technical and economic efficiency of medical diagnostic laboratories in hospitals affiliated with Urmia University of Medical Sciences (UUMS) in 2016.

\section{Methods}

In this descriptive-analytical study, the data of all diagnostic laboratories of the hospitals of UUMS were inputted into Frontier 4.1 software after taking the logarithm. Then, the technical and economic efficiency of the laboratories were obtained by estimating the production and cost function using the stochastic frontier analysis method, assuming input minimization for 2016.

\section{Results}

The average technical and economic efficiency of the diagnostic laboratories was obtained as $0.931 \pm 0.34$ and $0.519 \pm 0.33$, respectively. These laboratories must reduce their inputs and costs to achieve full efficiency without changing the amount of their output.

\section{Conclusions}

Although the average economic efficiency of the diagnostic laboratories of the studied hospitals was high, there is still an increase in the efficiency of these units given the cost of inputs at the time of allocating resources. In addition, it is possible to improve the technical efficiency of the clinical laboratories of hospitals affiliated with UUMS by $48.1 \%$ by applying the same level of inputs and without increasing the costs.

Key Words : hospital, diagnostic laboratory, Stochastic Frontier Analysis, economic efficiency

\section{Introduction}

Measuring the performance of hospitals' clinical laboratories as an important and costly unit is essential for their managers ${ }^{1}$ as they can demonstrate its strengths and weaknesses, identify areas that need improvement, and increase productivity in the hospital. ${ }^{2}$

A laboratory is a place where various operations such as empirical tests, various measurements, and the analysis and identification of materials and impurities are performed. ${ }^{3}$ In this regard, medical diagnostic laboratories play an important role in diagnosing and providing high-quality care to patients. ${ }^{4}$ Now that communities have recognized the value of health, it is not possible to maintain community health, prevent the spread of infectious diseases, and combat genetic diseases without the use of medical diagnostic laboratories. ${ }^{5}$

The laboratory is a highly sensitive and important work environment as it employs specialized labors in various fields as well as various costly equipment, and its performance and efficiency require a great deal of attention. ${ }^{6}$ In this regard, economic efficiency demonstrates the ability of the laboratory to obtain maximum benefit according to price and input levels. ${ }^{7}$ Economic efficiency is associated with a combination of technical efficiency and allocative efficiency. ${ }^{8}$ Technical efficiency indicates the ability of the laboratory to maximize the product (service delivery) with respect to specific production factors or to minimize production factors regarding the particular product. ${ }^{9}$ Allocation efficiency is also the allocation of limited resources to different inputs in order to maximize output. ${ }^{10}$

A laboratory has economic efficiency only if it is efficient both in the technical and allocational sense. That is, economic efficiency can be achieved if the best use is made of resources in the laboratory unit without wasting the resources. ${ }^{11}$ This requires a combination of institutions with the lowest cost; algebraically, economic efficiency is obtained by multiplying the quantities of technical and allocative efficiencies.

Different methods are used to evaluate the performance of service and manufacturing units. These methods are generally divided into nonparametric and parametric categories. ${ }^{12}$ One of the most common parametric methods is stochastic frontier analysis (SFA) in which statistical disturbances are considered, and it is necessary to take into account assumptions about the frontier form of the function. This method is an 
econometric technique that shows deviation from the best performance frontier and demonstrates the effect of the noise term on efficiency (such as device failure, measurement error, and strikes) which is beyond the control of production units. This feature divides the deviation of the frontier into two components of inefficiency and random error. ${ }^{13}$ In this method, the production function is estimated as the maximum product that can be produced from a set of production factors, and provides a better definition of inefficiency based on economic theory. The SFA seems to be an appropriate method if the rate of product factors and production is a random mechanism. ${ }^{14,15}$

The 2017 study by Alinejad et al. entitled "The economic efficiency of clinical laboratories in public hospitals: A case study in Iran" , the 2015 study by Taheri entitled "The efficiency of clinical laboratories affiliated with Shiraz University of Medical Sciences: an application of data envelopment analysis"16, and the 2017 study by Lamovsek entitled "Defining the optimal size of medical laboratories at the primary level of healthcare with data envelopment analysis: defining the efficiency of medical laboratories" ${ }^{4}$ have investigated the performance and types of efficiency of laboratories through data envelopment analysis and SFA.

Since studying the performance of hospitals' clinical laboratories and identifying their strengths and weaknesses are of great importance in the optimal allocation of facilities, the present study aimed to evaluate the efficiency of resource use in clinical laboratories of hospitals affiliated with Urmia University of Medical Sciences (UUMS) in 2017 via the SFA method. The results can help hospital managers and chiefs to improve the economic performance of laboratory units by avoiding the waste of scarce resources and thus reducing unit costs.

\section{Methods}

In this descriptive-analytical study, the technical and economic efficiency of 22 diagnostic laboratories of the hospitals affiliated with the UUMS was estimated using the SFA method by the variable returns to scale (VRS) and input-oriented assumptions via Frontier 4.1 software in 2017.

The general form of the Cobb-Douglas production function to calculate the technical efficiency of the laboratory units in this study is as follows ${ }^{17}$ :

$$
\begin{gathered}
\operatorname{Ln} Y_{\mathrm{it}}=\beta_{0}+\sum \beta_{j} X_{\mathrm{jit}}+\left(V_{\mathrm{it}}-U_{\mathrm{it}}\right) \\
\operatorname{Ln}\left(Y_{\mathrm{it}}\right)=\beta_{0}+\beta_{1} \operatorname{Ln}\left(P_{\mathrm{it}}\right)+\beta_{2} \operatorname{Ln}\left(E_{\mathrm{it}}\right)+\beta_{3} \operatorname{Ln}\left(T_{\mathrm{it}}\right)+\beta_{4} \operatorname{Ln}\left(I_{\mathrm{it}}\right)+\beta_{5} \operatorname{Ln}\left(S_{\mathrm{it}}\right)+\left(V_{\mathrm{it}}-U_{\mathrm{it}}\right)
\end{gathered}
$$

where Ln: logarithm at the base of natural number, Yit: production of unit i at time t, Xjit: rate of using factor $\mathrm{j}$ by unit $\mathrm{i}$ at time $\mathrm{t}$, Vit: random disturbance component, and Uit: model inefficiency. Inputs include the number of specialists $(\mathrm{P})$, experts $(\mathrm{E})$, technicians $(\mathrm{T})$, tools and equipment $(\mathrm{I})$, and materials and solutions (S), and the output contains the number of patients admitted to the laboratory unit $(\mathrm{Y})$.

The general form of the Cobb-Douglas cost function for estimating the economic efficiency of the laboratory units by the SFA method is as follows ${ }^{17}$ :

$\operatorname{Ln}\left(C_{\mathrm{it}} / W_{\mathrm{Pit}}\right)=\beta_{0}+\beta_{1} \operatorname{Ln}\left(Y_{\mathrm{it}}\right)+\beta_{2} \operatorname{Ln}\left(\frac{W_{\mathrm{Eit}}}{W_{\mathrm{Pit}}}\right)+\beta_{3} \operatorname{Ln}\left(\frac{W_{\mathrm{Tit}}}{W_{\mathrm{Pit}}}\right)+\beta_{4} \operatorname{Ln}\left(\frac{W_{\mathrm{Iit}}}{W_{\mathrm{Pit}}}\right)+\beta_{5} \operatorname{Ln}\left(\frac{W_{\text {Sit }}}{W_{\mathrm{Pit}}}\right)+\left(V_{\mathrm{it}}-U_{\mathrm{it}}\right)$

where Ln: logarithm at the base of natural number, Cit: total cost, Yit: number of patients admitted, WPit: specialist wages, WEit: expert wages, WTit: technician wages, WIit: the price of tools and equipment, WSit: the price of materials and solutions, Vit: random disturbance component, and Uit: model inefficiency. To calculate the total cost, the costs of properties (including medical and non-medical), construction, consumable, equipment and salaries of all employees in the laboratory unit, which are a valid representation of the total cost of that unit, were used. Moreover, part of the costs of the laboratory unit is the overhead costs that are split or shared between different parts of the hospital. 
The price of the equipment is actually its annual depreciation expense, and the straight line method was used to calculate the depreciation of equipment.

Also, in the SFA method, the numbers obtained for economic efficiency were divided into the highest economic efficiency figure until the most efficient laboratory unit acquired number 1 and the residual units fell $<1$; this allowed for the easy comparison of the technical and economic efficiency of efficient and inefficient units. In this case, the most efficient laboratory unit equaled one in technical and economical terms, and the rest were sub-one. ${ }^{18}$

\section{Results}

Table 1 summarizes the results of the stochastic frontier cost function estimation in Frontier 4.1. Since the index of the likelihood ratio (LR) test was high in this estimate for the Cobb-Douglas function, there was no need to estimate the translog function. In order to calculate the economic efficiency, it is necessary to estimate the cost function through the cost of inputs and the total cost of the laboratory unit. In this study, the total costs of construction, consumables, property, equipment, and staff salaries of the relevant laboratory were used to calculate the total cost of the hospitals' laboratory units.

Table 1 : Estimation of Frontier Cost Function Parameters (SFA) from the Maximum Likelihood Method (ML)

In Column 2 of Table 2, the amount of economic efficiency obtained from the cost function estimation is greater than one, because Frontier 4.1 does not consider a constraint in cost function estimation such as the economic efficiency range between zero and one. In order to compare the calculated SFA efficiencies, the economic efficiency figures obtained from the SFA method were divided into the highest cost efficiency figure in Table 2 (1.124) until the numerical values of economic efficiency fall between zero and one (Column 3 in Table 1). In this method, the average economic efficiency of the laboratory units of hospitals affiliated with the UUMS was 0.931 ( $\mathrm{SD}=0.034)$. Also, the lowest cost-efficiency belonged to Hospital Laboratory 9 (value $=0.89$ ), and the highest cost efficiency was related to Hospital 13 (value $=1$ ).

Table 2 : Economic efficiency of clinical laboratories of hospitals affiliated with the UUMS through the SFA method

In Table 3, no significant relationship is observed between output, i.e. the number of admissions, with expert, ELISA, cell counters, incubators, centrifuges, microscopes, and microbial culture medium inputs. In other words, these inputs do not have a considerable impact on the output level, which may be due to the high similarity of data collected from hospitals' laboratories and, therefore, a reduced fluctuation between data and their low variance. Also, in Table 3, the sum of the partial elasticity coefficients of the inputs was 1.94 , so the return to scale among the laboratory units of the studied hospitals was ascending. The negative production elasticity of some production factors indicated that the laboratories under study were in the third and noneconomic stages of production in terms of applying these factors. Also, the production elasticity relative to specialist input was 3.82, which was greater than the other elasticities. This means that a $1 \%$ increase in this production factor leads to the highest increase in the return of laboratories by $3.82 \%$. According to the final results of the maximum likelihood estimation regarding the accuracy of using the Cobb-Douglas function, since the LR value was 7.93, the Cobb-Douglas function form was chosen. In other words, due to the high LR value of the Cob-Douglas function rather than the translog function, this form was suitable for the SFA for the studied laboratory units. The gamma variable that shows the contribution of inefficiency variance in the production function also equaled 1 with a standard error of 0.0000006 . That is, the share of random factors in the inefficiency of clinical laboratories in hospitals affiliated with the UUMS was equal to zero, and the observed inefficiency component had the major contribution. The significance of the $\gamma$ parameter confirms that inefficiency plays an important role in the model.

Table 3 : Estimation of stochastic frontier production function (SFA) parameters from the maximum likelihood method (ML)

According to the results of Table 4, the average technical efficiency of medical diagnostic laboratories affiliated 
with the UUMS was 0.519 ( $\mathrm{SD}=0.33$ ). According to the SFA model, the lowest technical efficiency belonged to Hospital 17 (value $=0.049$ ) and the highest technical efficiency belonged to Hospitals 2 and 3 (value=0.999).

Table 4 : Technical efficiency of the clinical laboratories of hospitals affiliated with the UUMS through the SFA method

\section{Discussion}

The technical and economic efficiency of medical diagnostic laboratories of hospitals affiliated with the UUMS was estimated via SFA under VRS and input-oriented minimization assumption by estimating production and cost functions in order to evaluate their performance.

The low technical efficiency of medical diagnostic laboratories of the hospitals indicated the high capacity of inputs in these units. While using the same level of inputs, these units can increase their outputs and thus their technical efficiency by $48.1 \%$ without any increase in costs.

The average economic efficiency of the above-mentioned units was 0.931 . Therefore, the efficiency of these units can be improved by reducing costs by $6.9 \%$ without decreasing the value of outputs by increasing the profit. In other words, the managers of these laboratories must reduce their costs by optimally combining and distributing inputs, while taking into account the prices of the used inputs to approach the profitability frontier.

The average economic efficiency of laboratory units was higher than the average technical efficiency, which demonstrates the high allocative efficiency of these units; that is, the combination of inputs in these units is appropriate. However, service providers in these units can make the most of this combination to increase service delivery without changing the amount of inputs in order to increase technical efficiency. Therefore, in order to reduce costs and increase their profits, the above-mentioned laboratory units should avoid wasting resources by enhancing the motivation of the staff and the awareness of the general principles of medical diagnostic laboratories.

Furthermore, medical diagnostic laboratories of university hospitals had an increasing return to scale. In other words, the coefficient of function or degree of homogeneity was $>1$; that is, if inputs are increased by $1 \%$, the output of laboratories will increase by more than $1 \%$. Therefore, the laboratories under study should increase their supply of services.

Alinejhad et al. in a study entitled "Evaluating the economic efficiency of clinical laboratories in Iranian public hospitals via DEA method" achieved the average economic efficiency of $0.676 .{ }^{1}$ The results of examining the economic efficiency of laboratory units in hospitals affiliated with the UUMS by using DEA and SFA methods showed that the average economic efficiency of the units differed in the two methods (0.255). This difference could be due to neglecting random effects, measurement errors, etc. in the DEA method. In general, however, the average economic efficiency of the studied units in the province is medium to high, indicating that in the studied units, it is possible to increase economic efficiency by reducing costs without affecting output values.

After examining the efficiency of Australian hospitals by using DEA and SFA, Mortimer et al. concluded that each method of efficiency measurement has its own characteristics, and there is no single method for measuring the efficiency whose results would be more precise and reliable compared to other methods. ${ }^{13}$

In a study entitled "Analyzing the efficiency changes of Irish hospitals", Gannon concluded that the technical efficiency values obtained from the DEA method were higher than the SFA method; that is, the inefficiency in the DEA method was underestimated. ${ }^{19}$

In a study entitled "Evaluating the economic efficiency of CCU in hospitals affiliated with Tehran University of Medical Sciences", Zahavi et al. used both SFA and DEA methods and concluded that these two methods do not necessarily have the same results in calculating efficiency, and greater efficiency in one of these methods is not sustainable for all sectors. ${ }^{20}$ 
Lamovsek et al. determined the optimal size of medical laboratories at the primary healthcare level by using DEA. The average technical efficiency of the laboratories with the VRS assumption was obtained as 0.9333. The authors concluded that increasing the automation and consolidation of laboratory activities can affect the efficiency of laboratories and, thus, costs. ${ }^{4}$

In evaluating the technical efficiency of the clinical laboratories of Shiraz University of Medical Sciences, Taheri et al. reported the average technical efficiency of laboratory units to be $0.924^{16}$, which indicates a high level of technical efficiency in hospital units of Shiraz compared to Urmia.

In general, SFA is a useful tool for analyzing the efficiency of medical diagnostic laboratories, and its information may be useful for policy-making and performance evaluation of these units. The DEA and SFA methods are also complementary in efficiency measurement, but for efficiency measurement, it is recommended that the SFA method be first performed in order to identify the variables having a negative effect and to exclude them from the model.

One of the limitations of this study was the lack of participation of the private sector diagnostic laboratories. Better analysis and conclusions could be obtained from the efficiency status of provincial laboratories had these laboratories cooperated, and the health planners in the province could have used better data for decision making.

\section{CONCLUSION}

The findings of this study can provide a tool for better policy-making in clinical laboratories by helping the management of laboratory resources as a costly unit of the healthcare system. In addition, performance evaluation allows inefficient hospitals to compare their performance with that of similar but efficient hospitals and identify their promotion capabilities.

\section{ACKNOWLEDGEMENTS}

This article is part of a master's thesis with the IR.UMSU.REC.1397.160 code of ethics at Urmia University of Medical Sciences. The authors offer their sincere gratitude to the deputies of treatment and research of said university.

\section{CONFLICTS OF INTEREST}

No potential conflicts of interest relevant to this article were reported.

\section{REFERENCES}

1. Alinejhad M, Aghlmand S, Feizollahzadeh S, Yusefzadeh H. The economic efficiency of clinical laboratories in public hospitals: A case study in Iran. Journal of clinical laboratory analysis. 2019:e23067.

2. Arah OA, Klazinga NS, Delnoij DM, Asbroek At, Custers T. Conceptual frameworks for health systems performance: a quest for effectiveness, quality, and improvement.International journal for quality in health care.2003;15(5):377-398.

3. Ketabi S, Ahmadi-Ahwaz N, Moazzam E, Mobasherizadeh S, Alizadeh V. Efficiency evaluation of medical diagnostic laboratories using data envelopment analysis in Isfahan, Iran. Medical Laboratory Journal. 2015;9(3):102-109.

4. Lamovsek N, Klun M, Skitek M, Bencina J. Defining the Optimal Size of Medical Laboratories at the Primary Level of Health Care with Data Envelopment Analysis: Defining the Efficiency of Medical Laboratories. Acta Informatica Medica.2019;27(4):224.

5. Pagana KD, Pagana TJ. Mosby's Manual of Diagnostic and Laboratory Tests-E-Book. Elsevier Health Sciences; 2017.

6. Hudson J. Principles of clinical laboratory management: a study guide and workbook. Pearson Prentice Hall; 2004. 
7. Hollingsworth B. The measurement of efficiency and productivity of health care delivery. Health economics. 2008;17(10):1107-1128.

8. Murillo-Zamorano LR. Economic efficiency and frontier techniques. Journal of Economic surveys.2004;18(1):33-77.

9. Athanassopoulos A, Gounaris C. Assessing the technical and allocative efficiency of hospital operations in Greece and its resource allocation implications. European Journal of Operational Research. 2001;133(2):416431.

10. Yusefzadeh H, Ghorbani A, Lotfi F. Efficiency and productivity in health system. Yuban Publishing, First Edition, Tehran. 2017.

11. Peacock S, Chan C, Mangolini M, Johansen D. Techniques for measuring efficiency in health services.Productivity Commission: Staff Working Paper. 2001.

12. Kujawska J. Measurement of healthcare system efficiency in OECD countries. Metody Ilościowe w Badaniach Ekonomicznych. 2015;16(2):23-32.

13. Mortimer D, Peacock S.Hospital efficiency measurement: simple ratios vs frontier methods. Citeseer; 2002 .

14. Emami Meibodi A. Efficiency and productivity measurement (in theory and practice). Institute for trade studies and research press, Tehran, Iran.[In Persian]. 2006.

15. Worthington AC. Frontier efficiency measurement in health care: a review of empirical techniques and selected applications. Medical care research and review.2004;61(2):135-170.

16. Taheri A, Jahromi SAS, Lotfi F. Efficiency of Clinical Laboratories Affiliated Shiraz University of Medical Sciences in 2015: an Application of Data Envelopment Analysis.International Journal of Health Studies. 2017;2(4).

17. Coelli TJ. A guide to FRONTIER version 4.1: a computer program for stochastic frontier production and cost function estimation. CEPA Working papers;1996.

18. Aigner D, Lovell CK, Schmidt P. Formulation and estimation of stochastic frontier production function models. Journal of econometrics. 1977;6(1):21-37.

19. Gannon B. Testing for variation in technical efficiency of hospitals in Ireland. The Economic and Social Review. 2005;36(3):273-294.

20. Zahavi M, Arab M, GOUDARZI GR, Hoseini M, Akbarisari A, Akbarihaghighi F. Measurement of economic efficiency of CCUS of Tehran university of medical sciences hospitals, using Data Envelopment Analysis (DEA) and Stochastic Frontier Analysis (SFA) methods: 2006-2009. 2014.

\section{Hosted file}

Tables.docx available at https://authorea.com/users/331403/articles/458070-the-stochasticfrontier-analysis-technique-in-measuring-the-technical-and-economic-efficiency-ofhospital-diagnostic-laboratories-a-case-study-in-iran 